Received: 2018.06.03

Accepted: 2018.07.09

Published: 2018.08.16

\title{
A Predictive Risk Scoring System for Clinically Relevant Pancreatic Fistula After Pancreaticoduodenectomy
}

Authors' Contribution: Study Design A Data Collection B Statistical Analysis C Data Interpretation D Manuscript Preparation E Literature Search F Funds Collection G

\author{
BC 1,2 Wuzheng Xia* \\ BC 2 Yu Zhou* \\ BC 2 Ye Lin* \\ BF 2 Min Yu \\ BDF 2 Zi Yin \\ F 2 Xin Lu \\ F 2 Baohua Hou \\ AE 1,2 Zhixiang Jian
}

1 Southern Medical University, Guangzhou, Guangdong, P.R. China 2 Department of General Surgery, Guangdong General Hospital, Guangdong Academy of Medical Sciences, Guangzhou, Guangdong, P.R. China
Corresponding Author: Source of support:

* These authors contributed equally to this paper

Zhixiang Jian, e-mail: jianzx_118@163.com

This work was supported by grants from the National Natural Science Foundation of China (No. 81602172)

Background:

Material/Methods:

Results:

Conclusions:

\section{MeSH Keywords:}

Abbreviations:

Full-text PDF:
Postoperative pancreatic fistula remains a challenge after pancreaticoduodenectomy (PD). This study aimed to establish a scoring system to predict clinically relevant postoperative pancreatic fistula (CR-POPF) after PD. The clinical records of 361 consecutive patients who underwent PD between 2009 and 2017 were reviewed retrospectively. Patients were divided into a study group (225 patients) and a validation group (136 patients). CR-POPF was defined and classified based on the 2016 ISGPS definition and classification system. Univariate and multivariate logistic regression analyses were performed and we thus developed a scoring system based on the regression coefficient of the multivariate logistic regression model. The predictive value was determined using the receiver operating characteristic (ROC) curve.

A predictive scoring system with a maximum of 6 points for CR-POPF was established using the following 4 factors: 1 point for soft pancreatic texture $(\mathrm{OR} 2.09,95 \% \mathrm{Cl} 1.10-3.98, \mathrm{P}=0.025), 1.5$ points for main pancreatic duct diameter $\leq 2.5 \mathrm{~mm}(\mathrm{OR} 2.72,95 \% \mathrm{Cl} 1.23-5.99, \mathrm{P}=0.013), 0.5$ points for extended lymphadenectomy (OR $1.57,95 \% \mathrm{Cl} 1.13-2.18, \mathrm{P}=0.007), 0.5$ points for a $25-30 \mathrm{~g} / \mathrm{L}$ postoperative day 1 serum albumin $(\mathrm{OR} 1.43,95 \% \mathrm{Cl}$ $1.02-2.00, \mathrm{P}=0.037)$, and 3 points for postoperative day 1 serum albumin $\leq 25 \mathrm{~g} / \mathrm{L}(\mathrm{OR} 5.12,95 \% \mathrm{Cl} 1.82-14.41$, $\mathrm{P}=0.002$ ). The ROC curve showed that this scoring system was highly predictive for CR-POPF in the validation group (AUC=0.806, 95\%Cl: 0.735-0.878).

This 6-point risk scoring system will be useful for perioperative risk management of CR-POPF.

\section{Pancreatic Fistula • Pancreaticoduodenectomy • Risk Assessment}

POPF - postoperative pancreatic fistula; CR-POPF - clinically relevant postoperative pancreatic fistula; POD - postoperative day; Alb - albumin; OR - odds ratio; CI - confidence interval; ROC - receiver operating characteristic; AUC - area under the curve

https://www.medscimonit.com/abstract/index/idArt/911499 


\section{Background}

Pancreaticoduodenectomy (PD) is associated with high postoperative morbidity. Despite improvement in surgical techniques, instruments, and perioperative management, postoperative pancreatic fistula (POPF) still remains the major complication and a challenging event in patients undergoing PD [1]. POPF often leads to increased postoperative morbidity, prolonged hospital stays, increased medical costs, and sometimes to lifethreatening complications such as massive abdominal hemorrhages. Despite significant efforts in preventing this problem, the rate of POPF remains essentially unchanged $[2,3]$.

A uniform standard and definition for perioperative complications is crucial for surgical research. The International Study Group for Pancreatic Fistula (ISGPF) first proposed a standardized definition for POPF in 2005 [4]. In the past decade, some risk factors for POPF has been identified according to the 2005 grading system, such as sex, body mass index, status of the pancreatic parenchyma, diameter of the main pancreatic duct, kind of diseases, and bile juice infection [5,6]. However, despite the widespread use of the 2005 classification in the majority of published studies, several limitations still exist. For example, the definition of grade A POPF was considered too broad and lacks clinical consequences. The reported rates of grade A POPFs in the published literatures range from almost $0 \%$ to $50 \%$, suggesting its rate was either over- or under-estimated in past researches [7,8]. Additionally, there is a lack of clear classification of POPF as grade B or $\mathrm{C}$ when requiring an "invasive procedure" due to the presence of a "grey area" in the 2005 classification regarding whether the presence of some clinical procedures should move the grade of POPF from grade $\mathrm{B}$ to grade $\mathrm{C}[9,10]$.

In order to clarify and further refine the classification, the ISGPF revised the 2005 POPF definition and published the 2016 classification and grading of POPF [11]. A new concept of "biochemical leak (BF)" replaced the former grade A POPF. The grade A POPF is no longer considered a true pancreatic fistula because it has no clinical importance. In addition to a more accurate distinction between the grades B and C, the new 2016 classification stresses that a true POPF should have an impact on the clinical invention and outcome of the patient. To be defined strictly as a POPF, this condition needs to be clinically relevant. A clinically relevant postoperative pancreatic fistula (CR-POPF) is now redefined as a drain output of any measurable volume of fluid with an amylase level $>3$ times the upper limit of institutional normal serum amylase activity, associated with a clinically relevant development/condition related directly to the postoperative pancreatic fistula [11].

Recent large-scale studies clarified the reliability of the new definition and revealed it could better stratify distinct conditions that differ in clinical and economic outcomes [12,13]. However, there have been no reports on the risk factors related to CRPOPF based on the new definition. The aim of the present study was to identify perioperative risk factors of CR-POPF in a retrospective cohort of 225 patients undergoing pancreaticoduodenectomy in a single center.

\section{Material and Methods}

\section{Patients and data}

We retrospectively examined our prospectively maintained database of 361 consecutive patients who underwent PD at Guangdong General Hospital between January 01, 2009 and December 31, 2017. The 225 consecutive patients who underwent PD between January 01, 2009 and November 31, 2015 were assigned to the study group and the other 136 consecutive patients who underwent PD between December 01, 2015 and December 31, 2017 were assigned to the validation group.

The primary outcome, CR-POPF, was defined in accordance with the updated 2016 ISGPF consensus guidelines. Postoperative outcomes were analyzed with regards to POPF occurrence and its management, organ failure, mortality through 90 days after the operation, the occurrence of major surgical complications [Clavien-Dindo (CD) >3], postoperative mortality, length of hospital stay, and overall hospitalization costs. Demographic and preoperative clinical data were collected and were used to construct a predictive scoring model to predict the risk of CRPOPF after PD. The data obtained from the validation group were used for the internal validation of the predictive scoring model. The study was approved by the Medical Ethics Review Committee of Guangdong General Hospital.

\section{Surgical procedures for PD}

There were 7 surgeons who performed PD during this period, and surgical technique was largely performed by standard procedures. Generally, the operation was performed by using either classic PD or pylorus-preserving PD (PPPD) techniques. End-to-side, mucosa-mucosa anastomosis was the first choice for pancreaticojejunostomy. The pancreatic duct stent was not routinely used. In cases of very narrow pancreatic duct, or if it was impossible to expose the pancreas, an end-to-end pancreaticojejunostomy was performed. Usually, 3 abdominal drainage tubes were applied. One drainage tube was placed near the site of bile duct anastomosis, and the other 2 tubes were placed near the site of the pancreatic anastomosis. The techniques used primarily included both the classic PD and PPPD. During 2009-2015, the pancreatic resection volume remained stable. During the study period, there was a transition from classic Whipple operations to PPPD. 
Table 1. Patient characteristics for 361 patients underwent pancreaticoduodenectomy.

\begin{tabular}{|ccc}
\hline & N & \% \\
\hline Age (years, mean \pm sd.) & $58.2 \pm 12.2$ & \\
\hline Gender & & \\
\hline Male & 216 & 59.8 \\
\hline Female & 145 & 40.2 \\
\hline Disease & & \\
\hline Pancreatic ductal adenocarcinoma & 92 & 25.5 \\
\hline Bile duct cancer & 22 & 6.1 \\
\hline Ampullary cancer & 102 & 28.3 \\
\hline Duodenal neoplasm & 62 & 17.2 \\
\hline IPMN & 5 & 1.4 \\
\hline PNET & 23 & 6.4 \\
\hline Pancreatic serous cystadenoma & 6 & 1.7 \\
\hline Papillary mucinous cystadenoma & 3 & 0.8 \\
\hline Chronic pancreatitis & 16 & 4.4 \\
\hline Others & 30 & 8.3 \\
\hline Pathology & 308 & 85.3 \\
\hline Malignant & 53 & 14.7 \\
\hline Benign & & \\
\hline
\end{tabular}

sd - standard deviation; IPMN - intraductal papillary mucinous neoplasm; PNET - pancreatic neuroendocrine tumors.

\section{Statistical analysis}

Statistical analyses were performed using SPSS Statistics for Windows version 17.0 (IBM Corp., Armonk, NY) and R for Windows version 3.4.1. Categorical variables were analyzed using the Chi-squared test and continuous variables were analyzed using the $t$ test. Multivariate analysis was done for all variables with $P$ values of less than 0.1 by univariate analysis with a logistic regression analysis. Receiver operating characteristic (ROC) curves and the corresponding area under the curve (AUC) were used to evaluate how the prediction model performed on the test data. A P value of less than 0.05 was considered statistically significant.

\section{Results}

\section{Patients' characteristics}

Table 1 summarizes the demographic character of all 361 patients. The mean age of included patients was $58.2 \pm 12.2$ years,
$59.8 \%$ were male, and $40.2 \%$ were female. One hundred ninety-one (85.3\%) patients underwent pancreaticoduodenectomy for malignant diseases and 34 (14.7\%) patients underwent pancreaticoduodenectomy for benign diseases. Serious (grades III-V) complications occurred in 101 patients (28.0\%). Clinically relevant pancreatic fistulas (CR-POPF) were documented in 40 (POPF grade $B$ occurred in 30 and POPF grade $C$ occurred in 10) patients in the study group and in 21 (POPF grade $B$ occurred in 16 and POPF grade $C$ occurred in 5) patients in the validation group.

\section{Univariate and multivariate analyses of clinical variables in relation to CR-POPF after pancreaticoduodenectomy}

Table 2 lists the relationship between perioperative clinical variables and CR-POPF after pancreaticoduodenectomy based on the data of the study group. In univariate analysis, the main pancreatic duct diameter (MPD) $(P=0.008)$, soft pancreas $(P=0.019)$, intraoperative blood loss $(P=0.011)$, extended lymphadenectomy $(P=0.022)$, and serum albumin of the first postoperative day (postoperative day 1 albumin) $(P=0.001)$ were significantly higher in patients with CR-POPF. The following factors were entered into the multivariate analysis since the $P$ values were found to be less than 0.1 in univariate analysis: the MPD, pancreas thickness, pancreas texture, intraoperative blood loss, extended lymphadenectomy, and postoperative day 1 albumin. As shown in Table 3, multivariate logistic regression analysis identified pancreas texture (soft versus hard: OR $2.09,95 \% \mathrm{Cl} 1.10-3.98, \mathrm{P}=0.025$ ), MPD ( $\leq 2.5 \mathrm{~mm}$ versus $>2.5 \mathrm{~mm}$, OR $2.72,95 \% \mathrm{Cl} 1.23-5.99, \mathrm{P}=0.013$ ), extended lymphadenectomy (Yes versus No, OR $1.57,95 \% \mathrm{Cl}$ $1.13-2.18, P=0.007)$, and postoperative day 1 serum albumin $(\leq 25 \mathrm{~g} / \mathrm{L}$ versus $>30 \mathrm{~g} / \mathrm{L}$, OR $5.12,95 \% \mathrm{Cl} 1.82-14.41, \mathrm{P}=0.002$; $25-30 \mathrm{~g} / \mathrm{L}$ versus $>30 \mathrm{~g} / \mathrm{L}, \mathrm{OR} 1.43,95 \% \mathrm{Cl} 1.02-2.00, \mathrm{P}=0.037)$ were independent risk factors for CR-COPF.

The logistic regression model provided the estimated probability of CR-POPF after pancreaticoduodenectomy. This probability was equal to $y=1 /\left(1+e^{-z}\right)$, where $e$ is the base value of natural logarithms (a mathematical constant, $\approx 2.718281828459$ ), and $Z=-5.367+0.992 \times$ pancreas texture $(0$, hard pancreas; 1 , soft pancreas $)+1.421 \times$ MPD $(0,>2.5 \mathrm{~mm} ; 1, \leq 2.5 \mathrm{~mm})+0.587 \times \mathrm{ex}-$ tended lymphadenectomy (0,No; 1, Yes) $+(3.87 \times$ albumin $(0,>30$ $\mathrm{g} / \mathrm{L} ; 1, \leq 25 \mathrm{~g} / \mathrm{L})$ or $0.419 \times$ albumin $(0,>30 \mathrm{~g} / \mathrm{L} ; 1,25-30 \mathrm{~g} / \mathrm{L}))$.

We developed a score using variables based on the regression coefficient of the logistic regression model (Table 4). The equation for the scoring system was calculated on the assumption that a patient receives 1 point for a soft pancreas, 1.5 points for MPD $\leq 2.5 \mathrm{~mm}, 0.5$ points for extended lymphadenectomy, 3 points for postoperative day 1 albumin $\leq 25 \mathrm{~g} / \mathrm{L}$, and 0.5 points for albumin $25-30 \mathrm{~g} / \mathrm{L}$. The predictive value of this scoring system was also assessed in a validation group. The comparison 
Table 2. Univariate analyses of factors associated with CR-POPF after pancreaticoduodenectomy in the study group.

\begin{tabular}{|c|c|c|c|}
\hline Factor & $\begin{array}{l}\text { Patients without } \\
\text { CR-POPF }(n=185)\end{array}$ & $\begin{array}{l}\text { Patients with } \\
\text { CR-POPF }(n=40)\end{array}$ & $P$ value \\
\hline Age (years, mean \pm sd.) & $57.7 \pm 12.5$ & $60.2 \pm 9.8$ & 0.245 \\
\hline Gender & & & 0.801 \\
\hline Male & 107 & 24 & \\
\hline Female & 78 & 16 & \\
\hline History of diabetes & & & 0.376 \\
\hline Yes & 15 & 5 & \\
\hline No & 170 & 35 & \\
\hline Jaundice & & & 0.158 \\
\hline Yes & 100 & 30 & \\
\hline No & 85 & 10 & \\
\hline \multicolumn{4}{|l|}{ ASA-PS } \\
\hline 1 & 57 & 9 & \\
\hline 2 or 3 & 128 & 31 & \\
\hline Disease & & & 0.341 \\
\hline Malignant & 159 & 32 & \\
\hline Benign & 26 & 8 & \\
\hline Relation to portal vein on $\mathrm{CT}$ & & & 0.271 \\
\hline Involved portal vein & 17 & 6 & \\
\hline Away from portal vein & 168 & 34 & \\
\hline MPD diameter ( $\mathrm{mm}$, mean $\pm \mathrm{sd}$.) & $3.65 \pm 2.36$ & $2.70 \pm 1.60$ & 0.008 \\
\hline Pancreas thickness (mm, mean \pm sd.) & $13.10 \pm 1.36$ & $13.92 \pm 1.71$ & 0.081 \\
\hline Pancreas texture & & & 0.019 \\
\hline Soft & 65 & 22 & \\
\hline Hard & 120 & 18 & \\
\hline Blood loss (ml, mean \pm sd.) & $355.83 \pm 390.73$ & $589.66 \pm 520.54$ & 0.011 \\
\hline Operation time (min, mean \pm sd.) & $426.21 \pm 106.82$ & $439.74 \pm 99.57$ & 0.261 \\
\hline Procedure & & & 0.837 \\
\hline $\mathrm{PD}$ & 21 & 5 & \\
\hline PPPD & 164 & 35 & \\
\hline Extended lymphadenectomy & & & 0.022 \\
\hline Yes & 38 & 15 & \\
\hline No & 147 & 25 & \\
\hline Pncreaticojejunostomy & & & 0.360 \\
\hline Duct-to-mucosa & 121 & 22 & \\
\hline
\end{tabular}

This work is licensed under Creative Common Attribution NonCommercial-NoDerivatives 4.0 International (CC BY-NC-ND 4.0)

\section{2}

Indexed in: [Current Contents/Clinical Medicine] [SCI Expanded] [ISI Alerting System] [ISI Journals Master List] [Index Medicus/MEDLINE] [EMBASE/Excerpta Medica] [Chemical Abstracts/CAS] 
Table 2 continued. Univariate analyses of factors associated with CR-POPF after pancreaticoduodenectomy in the study group.

\begin{tabular}{|c|c|c|c|}
\hline Factor & $\begin{array}{l}\text { Patients without } \\
\text { CR-POPF ( } n=185 \text { ) }\end{array}$ & $\begin{array}{l}\text { Patients with } \\
\text { CR-POPF (n=40) }\end{array}$ & $P$ value \\
\hline Dunking method & 57 & 15 & \\
\hline Others & 7 & 3 & \\
\hline Pancreatic duct stent & & & 0.345 \\
\hline Internal stent & 64 & 17 & \\
\hline None & 121 & 23 & \\
\hline Preoperative CA19-9 & $180.56 \pm 289.46$ & $192.91 \pm 269.35$ & 0.805 \\
\hline Preoperative CA125 & $20.52 \pm 19.86$ & $17.06 \pm 10.24$ & 0.310 \\
\hline Preoperative CEA & $4.92 \pm 11.36$ & $5.69 \pm 10.20$ & 0.691 \\
\hline \multicolumn{4}{|l|}{ Albumin } \\
\hline Preoperative & $32.9 \pm 6.1$ & $32.7 \pm 4.8$ & 0.898 \\
\hline Postoperative Day 1 & $25.9 \pm 4.96$ & $23.8 \pm 4.04$ & 0.001 \\
\hline \multicolumn{4}{|l|}{ Total bilirubin } \\
\hline Preoperative & $147.69 \pm 141.36$ & $169.06 \pm 143.43$ & 0.389 \\
\hline Postoperative Day 1 & $124.24 \pm 126.96$ & $155.92 \pm 105.26$ & 0.143 \\
\hline \multicolumn{4}{|l|}{ Direct bilirubin } \\
\hline Preoperative & $83.01 \pm 79.77$ & $96.24 \pm 78.33$ & 0.347 \\
\hline Postoperative Day 1 & $68.36 \pm 73.56$ & $82.82 \pm 62.15$ & 0.250 \\
\hline \multicolumn{4}{|l|}{ Hemoglobin } \\
\hline Preoperative & $119.34 \pm 19.04$ & $120.18 \pm 18.66$ & 0.799 \\
\hline Postoperative Day 1 & $114.66 \pm 75.98$ & $105.69 \pm 14.02$ & 0.460 \\
\hline \multicolumn{4}{|l|}{ White blood cell } \\
\hline Preoperative & $7.16 \pm 2.59$ & $6.99 \pm 2.08$ & 0.696 \\
\hline Postoperative Day 1 & $13.26 \pm 4.40$ & $13.80 \pm 4.91$ & 0.498 \\
\hline \multicolumn{4}{|l|}{ Platelet } \\
\hline Preoperative & $278.62 \pm 93.00$ & $297.58 \pm 99.73$ & 0.251 \\
\hline Postoperative Day 1 & $251.36 \pm 93.69$ & $262.91 \pm 101.69$ & 0.488 \\
\hline \multicolumn{4}{|l|}{ Blood glucose } \\
\hline Preoperative & $6.16 \pm 2.42$ & $5.92 \pm 1.54$ & 0.545 \\
\hline Postoperative Day 1 & $10.97 \pm 10.05$ & $11.09 \pm 4.65$ & 0.941 \\
\hline \multicolumn{4}{|l|}{ PT } \\
\hline Preoperative & $14.22 \pm 1.51$ & $12.81 \pm 1.81$ & 0.447 \\
\hline Postoperative Day 1 & $14.68 \pm 1.32$ & $13.27 \pm 1.92$ & \\
\hline
\end{tabular}

sd - standard deviation; BMI - body mass index; PT - prothrombin time; $\mathrm{Na}$ - natrium; $\mathrm{K}$ - kalium; Mg - magnesium; Ca - calcium; MPD - main pancreatic duct diameter; ASA-PS - American Society of Anesthesiologists-physical status. 
Table 3. Results of multivariate analysis of factors predicting CR-POPF.

\begin{tabular}{|lcccc}
\multicolumn{1}{c}{ Variable } & \multicolumn{1}{c}{ Category } & OR & 95\%Cl & P value \\
\hline Pancreas texture & Soft versus Hard & 2.09 & $1.10-3.98$ & 0.025 \\
\hline MPD & $\leq 2.5 \mathrm{~mm}$ versus $>2.5 \mathrm{~mm}$ & 2.72 & $1.23-5.99$ & 0.013 \\
\hline Extended lymphadenectomy & Yes versus No & 1.57 & $1.13-2.18$ & 0.007 \\
\hline Postoperative day 1 serum ALB & $\leq 25 \mathrm{~g} / \mathrm{L}$ versus $>30 \mathrm{~g} / \mathrm{L}$ & 5.12 & $1.82-14.41$ & 0.002 \\
\hline Postoperative day 1 serum ALB & $25-30$ g/L versus $>30 \mathrm{~g} / \mathrm{L}$ & 1.43 & $1.02-2.00$ & 0.037 \\
\hline
\end{tabular}

MPD - main pancreatic duct diameter; ALB - albumin; Mg - magnesium.

Table 4. Score of each variable in the predictive scoring system for pancreatic fistula.

\begin{tabular}{|cc}
\hline \multicolumn{1}{c}{ Variables } & Points \\
\hline Pancreas texture & 1 \\
\hline Soft pancreas & 0 \\
\hline Hard pancreas & 1.5 \\
\hline MPD & 0 \\
\hline$\leq 2.5 \mathrm{~mm}$ & \\
\hline$>2.5 \mathrm{~mm}$ & 0.5 \\
\hline Extended lymphadenectomy & 0 \\
\hline Yes & \\
\hline No & 3 \\
\hline Postoperative day 1 albumin & 0.5 \\
\hline$\leq 25 \mathrm{~g} / \mathrm{L}$ & 0 \\
\hline $25-30$ g/L & \\
\hline$>30$ g/L &
\end{tabular}

between characteristics of patients are shown in Table 5 . As shown in Figure 1, the ROC curves based on the above scoring system show that the area under the curve (AUC) was 0.813 (95\%Cl: $0.737-0.889)$ in the study group (AUC1) and 0.806 (95\% Cl: $0.735-0.878)$ in the validation group (AUC2), respectively. The high AUC value in the validation group suggested that this scoring system was highly predictive of CR-POPF. Based on the sensitivities and specificities of every score, we concluded that a score $\geq 4.5$ could be used as a threshold to identify patients with high risk of developing CR-POPF.

\section{Discussion}

Postoperative PF (POPF) after pancreatic surgery remains a challenging event in high-volume pancreatic centers. Currently, a variety of risk factors related to POPF have been identified, such as sex, pancreatic texture, body mass index, and MPD. The 2016 new definition and grading of POPF by ISGPF highlighted the concept that the fistula must be a clinically relevant condition. Prediction of CR-POPF remains controversial, and many studies focused on preoperative factors but only few studies take into account postoperative factors. In the present study, we searched both preoperative and postoperative risk factors for the development of CR-POPF, and found that soft pancreatic texture, MPD, extended lymphadenectomy, and postoperative serum albumin were independent risk factors related to CR-POPF. These findings characterized a high-risk patient group for CR-POPF after pancreaticoduodenectomy.

Soft pancreatic texture and low serum albumin have been accepted as significant risk factors for POPF. In this study, both soft pancreas and postoperative serum albumin were identified as independent factors related to CR-POPF. Patients with a soft pancreas usually have a smaller pancreatic duct diameter and a larger parenchymal thickness than those with a hard pancreas. Histologic evaluation of soft pancreas showed a larger area of the cut surface, a smaller fibrosis ratio, and a larger lobular ratio.14 Decreased fibrosis in pancreatic tissue is associated with increased exocrine activity $[15,16]$ Additionally, a fatty pancreas might be more friable and is more easily disrupted during anastomosis than is a hard pancreas [17]. Relles et al. reported that hypoalbuminemia on postoperative day 1 was an independent predictive marker for complications after pancreaticoduodenectomy, which was consistent with our findings. Serum albumin is associated with nutritional status, and hypoalbuminemia is often associated with protein turnover and poor tissue healing, which attributes to anastomotic leakage [18-20].

Extended lymphadenectomy has not been investigated previously as a risk factor for PF after PD. In an earlier study, Yeo et al. [21] reported that periampullary adenocarcinoma patients undergoing radical pancreaticoduodenectomy (with the addition of a distal gastrectomy and extended retroperitoneal lymphadenectomy to a standard pancreaticoduodenectomy) had a significantly higher incidence of pancreatic fistula when comparing the standard 
Table 5. Comparison of the characters between the study group and the validation group.

\begin{tabular}{|c|c|c|c|}
\hline Factor & Patients without ( $n=225$ ) & Patients with $(n=136)$ & P value \\
\hline Age (years, mean \pm sd.) & $58.1 \pm 12.1$ & $58.3 \pm 12.5$ & 0.986 \\
\hline Gender & & & 0.422 \\
\hline Male & 131 & 85 & \\
\hline Female & 94 & 51 & \\
\hline History of diabetes & & & 0.273 \\
\hline Yes & 20 & 17 & \\
\hline No & 205 & 119 & \\
\hline Jaundice & & & 0.447 \\
\hline Yes & 130 & 73 & \\
\hline No & 95 & 63 & \\
\hline ASA-PS & & & 0.987 \\
\hline 1 & 66 & 40 & \\
\hline 2 or 3 & 159 & 96 & \\
\hline CR-POPF & & & 0.918 \\
\hline Grade B & 30 & 16 & \\
\hline Grade C & 10 & 5 & \\
\hline Disease & & & 0.767 \\
\hline Malignant & 191 & 117 & \\
\hline Benign & 34 & 19 & \\
\hline Relation to portal vein on $\mathrm{CT}$ & & & 0.282 \\
\hline Involved portal vein & 23 & 19 & \\
\hline Away from portal vein & 202 & 117 & \\
\hline MPD diameter (mm, mean \pm sd.) & $3.48 \pm 2.27$ & $3.50 \pm 2.63$ & 0.906 \\
\hline Pancreas thickness (mm, mean \pm sd.) & $13.25 \pm 1.46$ & $13.21 \pm 1.81$ & 0.879 \\
\hline Pancreas texture & & & 0.153 \\
\hline Soft & 87 & 63 & \\
\hline Hard & 138 & 73 & \\
\hline Blood loss (ml, mean \pm sd.) & $397.40 \pm 424.98$ & $367.25 \pm 390.77$ & 0.671 \\
\hline Operation time (min, mean $\pm s d$.) & $428.62 \pm 105.48$ & $409.46 \pm 112.58$ & 0.779 \\
\hline Procedure & & & 0.004 \\
\hline PD & 26 & 5 & \\
\hline PPPD & 199 & 131 & \\
\hline Extended lymphadenectomy & & & 0.868 \\
\hline Yes & 53 & 31 & \\
\hline No & 172 & 105 & \\
\hline
\end{tabular}


Table 5 continued. Comparison of the characters between the study group and the validation group.

\begin{tabular}{|c|c|c|c|}
\hline Factor & Patients without $(n=225)$ & Patients with $(n=136)$ & $P$ value \\
\hline Pncreaticojejunostomy & & & 0.001 \\
\hline Duct-to-mucosa & 143 & 131 & \\
\hline Dunking method & 72 & 0 & \\
\hline Others & 10 & 5 & \\
\hline Pancreatic duct stent & & & 0.163 \\
\hline Internal stent & 81 & 59 & \\
\hline None & 144 & 77 & \\
\hline \multicolumn{4}{|l|}{ Albumin } \\
\hline Preoperative & $32.9 \pm 5.9$ & $35.2 \pm 5.1$ & 0.023 \\
\hline Postoperative Day 1 & $25.5 \pm 4.9$ & $29.8 \pm 4.8$ & 0.001 \\
\hline \multicolumn{4}{|l|}{ Hemoglobin } \\
\hline Preoperative & $119.49 \pm 18.93$ & $121.36 \pm 19.52$ & 0.516 \\
\hline Postoperative Day 1 & $105.69 \pm 69.20$ & $111.66 \pm 21.37$ & 0.298 \\
\hline \multicolumn{4}{|l|}{ White blood cell } \\
\hline Preoperative & $7.13 \pm 2.50$ & $7.06 \pm 2.73$ & 0.812 \\
\hline Postoperative Day 1 & $13.36 \pm 4.49$ & $13.47 \pm 5.62$ & 0.701 \\
\hline \multicolumn{4}{|l|}{ Platelet } \\
\hline Preoperative & $281.99 \pm 94.28$ & $287.71 \pm 96.43$ & 0.834 \\
\hline Postoperative Day 1 & $253.41 \pm 95.03$ & $249.61 \pm 97.29$ & 0.912 \\
\hline
\end{tabular}

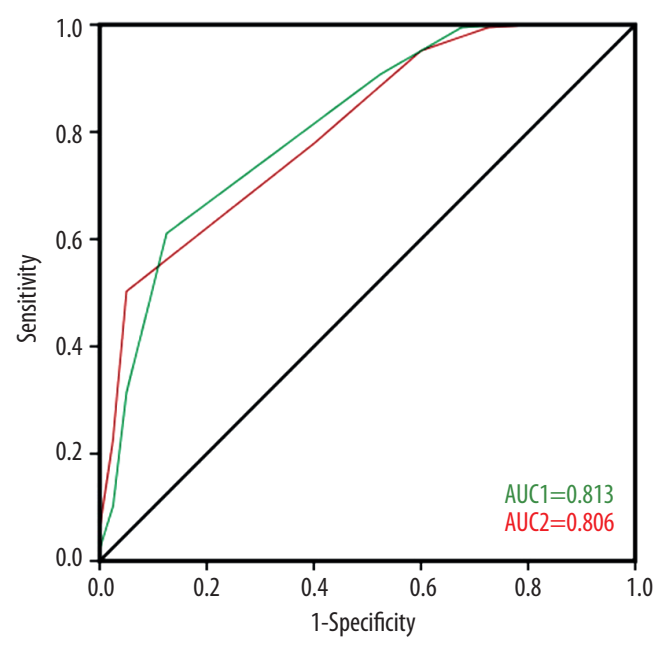

\begin{tabular}{cccccc}
\hline \multirow{2}{*}{ Scores } & \multicolumn{2}{c}{ AUC1: The study group } & & \multicolumn{2}{c}{ AUC2: The validation group } \\
\cline { 2 - 3 } \cline { 5 - 6 } & Sensitivity & Specificity & & Sensitivity & Specificity \\
\hline 0.5000 & 0.000 & 1.000 & & 1.000 & 0.000 \\
1.0000 & 1.000 & 0.000 & & 1.000 & 0.025 \\
1.5000 & 1.000 & 0.050 & & 1.000 & 0.075 \\
2.0000 & 1.000 & 0.075 & & 1.000 & 0.125 \\
2.5000 & 1.000 & 0.150 & & 1.000 & 0.200 \\
3.0000 & 1.000 & 0.250 & & 0.995 & 0.275 \\
3.5000 & 0.995 & 0.325 & & 0.951 & 0.400 \\
4.0000 & 0.908 & 0.475 & & 0.778 & 0.600 \\
4.5000 & 0.611 & 0.875 & & 0.503 & 0.950 \\
5.0000 & 0.314 & 0.950 & & 0.227 & 0.975 \\
5.5000 & 0.103 & 0.975 & & 0.059 & 1.000 \\
6.0000 & 0.022 & 1.000 & & 0.011 & 1.000 \\
\hline
\end{tabular}

Figure 1. Two receiver operating characteristic (ROC) curves for the estimated probability of CR-POPF calculated based on the scoring system. The area under the curve for the study group (AUC1) was 0.813 (95\% Cl: $0.737-0.889)$ and the area under the curve for the validating group (AUC2) was 0.806 (95\% Cl: 0.735-0.878). 
Table 6. Comparison between the present scoring system and existing scoring systems.

\begin{tabular}{|cccc}
\hline Score system & Scoring factors & Population & AUC* \\
\hline The present study & $9,10,12,13$ & Pancreatoduodenectomy & 0.806 \\
\hline Gaujoux et al. [24] & $4,14,15$ & Pancreatoduodenectomy & 0.801 \\
\hline Wellner et al. [25] & $1,3,5,6,7$ & Pancreatoduodenectomy & 0.656 \\
\hline Callery et al. [26] & $7,8,9,10$ & Pancreatoduodenectomy & 0.789 \\
\hline Roberts et al. [27] & 4,10 & Pancreatoduodenectomy & 0.702 \\
\hline Yamamoto et al. [28] & $2,7,10,11,16$ & Pancreatoduodenectomy & 0.734 \\
\hline Belyaev et al. [29] & 10,19 & Pancreatoduodenectomy, distal pancreatectomy & NA \\
\hline Nahm et al. [30] & 17 & $\begin{array}{l}\text { Pancreatoduodenectomy, distal pancreatectomy, central } \\
\text { pancreatectomy }\end{array}$ \\
\hline Nahm et al. [31] & 18 & Distal pancreatectomy \\
\hline
\end{tabular}

1 - age; 2 - gender; 3 - smoking history; 4 - BMI; 5 - weight loss; 6 -, pancreatitis history; 7 - preoperative diagnosis other than pancreatic cancer or chronic pancreatitis; 8 - blood loss; 9 - pancreatic texture; 10 - pancreatic duct diameter; 11 - relation of portal vein to tumor; 12 - extended lymphadenectomy; 13 - serum albumin of postoperative day 1; 14 - pancreatic fibrosis; 15 - presence of fatty pancreas, 16 - intra-abdominal fat thickness; 17 - acinar cell density at the pancreatic resection margin; 18 - intra-operative amylase concentration; 19 - histomorphological features including periductal fibrosis, interlobular fibrosis, intralobular fibrosis, interlobular fat, intralobular fat, signs of chronic pancreatitis, signs of acute pancreatitis, tissue edema. AUC - area under the curve; NA - result was not available due to lack of data. * AUC was obtained by the receiver operating characteristic (ROC) curve analysis in the validation group.

pancreaticoduodenectomy (13\% vs. $6 \% ; \mathrm{P}=0.05)$. A recent study reported that extended lymphadenectomy was an independent risk factor for clinical pancreatic fistula after distal pancreatectomy [22]. On the other hand, the high morbidity rate also was reported in patients with pancreatic body cancer who underwent extended lymphadenectomy.23 The higher rate of pancreatic fistula in the radical group also is not clearly explainable.

Several scoring systems have been reported before [24-28]. A comparison between the present scoring system and these existing scoring systems in term of CR-POPF based on the validation group is summarized in Table 6 . The systems by Yamamoto [28], Wellner [25], and Roberts [27] use easily measurable radiological and demographic variables and may be constructed preoperatively. Preoperative risk stratification has certain advantages, including the opportunity to individualize patient consent, to facilitate training opportunities in lowrisk patients, and to select optimal patients for clinical trials. However, due to the exclusion of intraoperative and postoperative factors, the predictive value of preoperative scoring systems might be compromised. Some studies included postoperative pathological factors in their scoring system. For example, Gaujoux et al. [24] proposed a predictive scoring system using BMI, pancreatic fibrosis, and the presence of fatty pancreas. Although their scoring system is quite useful for estimating POPF, it requires pathological examination; therefore, the final score can be delayed until several days after the operation. To address the risk of POPF accurately and quickly, we included preoperative and intraoperative factors, as well as factors on the first postoperative day. Additionally, unlike most other scoring systems, the present study just selected patients with "clinically relevant" pancreatic fistula (ISGPF types $B$ and $C)$, making our scoring system more clinically significant.

Additional scores have been proposed to predict POPF in patients undergoing a general pancreatic resection. Belyaev et al. [29] proposed a scoring system based on histomorphological features of the pancreatic remnant, and Nahm et al30 suggested that acinar cell density in the pancreatic resection margin was significantly associated with POFP for all pancreatic resections. Because the results of histomorphological features and acinar cell density depend on the pathological examination, the final score could be delayed until several days postoperatively, but these pathological parameters showed good predictive value in past studies; they warrant rapid pathological examinations if their predictive value can be firmly established by further studies. Another study, by Nahm et al. [31], reported that intraoperative amylase concentration was significantly correlated with POPF in patients who underwent distal pancreatectomy. Both intraoperative amylase concentration and acinar cell density are unconventional risk factors that were not evaluated in our center before. Further studies are needed to confirm their value in predicting CR-POPF alone or as part of a scoring system.

In the present study, the validation group had a significantly increased rate of PPPD procedure and proportion of 
duct-to-mucosa anastomosis. PPPD is now the first choice in our center, and we use duct-to-mucosa anastomosis as often as we can. The albumin level in the validation group was significantly higher than in the study group. This difference can be explained by the preoperative nutritional assessment and support. Since more than 2 years ago, we started to routinely assess nutritional status by using NRS2002 in patients who were planned to undergo pancreaticoduodenectomy, and the nutritional support treatment was performed in patients with NRS2002 score $\geq 3$. Despite the differences in baseline characteristics of patients in these 2 groups, the AUC in the validation group was 0.806 , suggesting the extrapolation of our scoring system is high.

\section{References:}

1. Malleo G, Pulvirenti A, Marchegiani G et al: Diagnosis and management of postoperative pancreatic fistula. Langenbecks Arch Surg, 2014; 399(7): 801-10

2. Ecker BL, MCMillan MT, Allegrini V et al: Risk factors and mitigation strategies for pancreatic fistula after distal pancreatectomy: Analysis of 2026 resections from the International, Multi-Institutional Distal Pancreatectomy Study Group. Ann Surg, 2017 [Epub ahead of print]

3. Shrikhande SV, D'Souza MA: Pancreatic fistula after pancreatectomy: Evolving definitions, preventive strategies and modern management. World J Gastroenterol, 2008; 14(38): 5789-96

4. Bassi C, Dervenis C, Butturini G et al: Postoperative pancreatic fistula: An international study group (ISGPF) definition. Surgery, 2005; 138(1): 8-13

5. Vallance $A E$, Young AL, Macutkiewicz $C$ et al: Calculating the risk of a pancreatic fistula after a pancreaticoduodenectomy: A systematic review. HPB (Oxford), 2015; 17(11): 1040-48

6. McMillan MT, Vollmer CM Jr. Predictive factors for pancreatic fistula following pancreatectomy. Langenbecks Arch Surg, 2014; 399(7): 811-24

7. Ho IG, Kim JK, Hwang HK et al: Does international study group on pancreatic fistula (ISGPF) classification need modification after distal pancreatectomy? Korean J Hepatobiliary Pancreat Surg, 2014; 18(3): 90-93

8. Gebauer F, Kloth K, Tachezy M et al: Options and limitations in applying the fistula classification by the International Study Group for Pancreatic Fistula. Ann Surg, 2012; 256(1): 130-38

9. Hackert T, Hinz U, Pausch T et al: Postoperative pancreatic fistula: We need to redefine grades B and C. Surgery, 2016; 159(3): 872-77

10. Fuks D, Piessen $G$, Huet $E$ et al: Life-threatening postoperative pancreatic fistula (grade C) after pancreaticoduodenectomy: Incidence, prognosis, and risk factors. Am J Surg, 2009; 197(6): 702-9

11. Bassi C, Marchegiani G, Dervenis C et al: The 2016 update of the International Study Group (ISGPS) definition and grading of postoperative pancreatic fistula: 11 years after. Surgery, 2017; 161(3): 584-91

12. Pulvirenti A, Marchegiani G, Pea A et al: Clinical implications of the 2016 International Study Group on Pancreatic Surgery definition and grading of postoperative pancreatic fistula on 775 consecutive pancreatic resections. Ann Surg, 2017 [Epub ahead of print]

13. Chen BP, Bennett S, Bertens KA et al: Use and acceptance of the International Study Group for Pancreatic Fistula (ISGPF) definition and criteria in the surgical literature. HPB (Oxford), 2018; 20(1): 69-75

14. Sugimoto $M$, Takahashi $S$, Kojima $M$ et al: In patients with a soft pancreas, a thick parenchyma, a small duct, and fatty infiltration are significant risks for pancreatic fistula after pancreaticoduodenectomy. J Gastrointest Surg, 2017; 21(5): 846-54

15. Friess $H$, Malfertheiner $P$, Isenmann $R$ et al: The risk of pancreaticointestinal anastomosis can be predicted preoperatively. Pancreas, 1996; 13(2): 202-8

16. Uchida E, Tajiri T, Nakamura Y et al: Relationship between grade of fibrosis in pancreatic stump and postoperative pancreatic exocrine activity after pancreaticoduodenectomy: Eith special reference to insufficiency of pancreaticointestinal anastomosis. J Nippon Med Sch, 2002; 69(6): 549-56

\section{Conclusions}

Despite these limitations, this study has developed a novel predictive scoring system for CR-POPF after PD, with good extrapolation ability. This scoring system may help surgeons to identify and control CR-POPF earlier, in order to improve the management of POPF and prevent more severe complications.

\section{Conflicts of interest}

None.

17. Mathur A, Pitt HA, Marine M et al: Fatty pancreas: A factor in postoperative pancreatic fistula. Ann Surg, 2007; 246(6): 1058-64

18. Relles DM, Richards NG, Bloom JP et al: Serum blood urea nitrogen and serum albumin on the first postoperative day predict pancreatic fistula and major complications after pancreaticoduodenectomy. J Gastrointest Surg 2013; 17(2): 326-31

19. Fujiwara $\mathrm{Y}$, Shiba $\mathrm{H}$, Shirai $\mathrm{Y}$ et al: Perioperative serum albumin correlates with postoperative pancreatic fistula after pancreaticoduodenectomy. Anticancer Res, 2015; 35(1): 499-503

20. Hennessey DB, Burke JP, Ni-Dhonochu T et al: Preoperative hypoalbumin emia is an independent risk factor for the development of surgical site infection following gastrointestinal surgery: A multi-institutional study. Ann Surg, 2010; 252(2): 325-29

21. Yeo CJ, Cameron JL, Lillemoe KD et al: Pancreaticoduodenectomy with or without distal gastrectomy and extended retroperitoneal lymphadenectomy for periampullary adenocarcinoma, part 2: randomized controlled trial evaluating survival, morbidity, and mortality. Ann Surg, 2002; 236(3): 35566; discussion 366-68

22. Yoshioka R, Saiura A, Koga R et al: Risk factors for clinical pancreatic fistula after distal pancreatectomy: analysis of consecutive 100 patients. World J Surg, 2010; 34(1): 121-25

23. Hirano S, Kondo S, Hara T et al: Distal pancreatectomy with en bloc celiac axis resection for locally advanced pancreatic body cancer: Long-term results. Ann Surg, 2007; 246(1): 46-51

24. Gaujoux S, Cortes A, Couvelard A et al: Fatty pancreas and increased body mass index are risk factors of pancreatic fistula after pancreaticoduodenectomy. Surgery, 2010; 148(1): 15-23

25. Wellner UF, Kayser $\mathrm{G}$, Lapshyn $\mathrm{H}$ et al: A simple scoring system based on clinical factors related to pancreatic texture predicts postoperative pancreatic fistula preoperatively. HPB (Oxford), 2010; 12(10): 696-702

26. Callery MP, Pratt WB, Kent TS et al: A prospectively validated clinical risk score accurately predicts pancreatic fistula after pancreatoduodenectomy. J Am Coll Surg, 2013; 216(1): 1-14

27. Roberts KJ, Hodson J, Mehrzad $\mathrm{H}$ et al: A preoperative predictive score of pancreatic fistula following pancreatoduodenectomy. HPB (Oxford), 2014; 16(7): 620-28

28. Yamamoto $\mathrm{Y}$, Sakamoto $\mathrm{Y}, \mathrm{Nara} \mathrm{S}$ et al: A preoperative predictive scoring system for postoperative pancreatic fistula after pancreaticoduodenectomy. World J Surg, 2011; 35(12): 2747-55

29. Belyaev O, Munding J, Herzog T et al: Histomorphological features of the pancreatic remnant as independent risk factors for postoperative pancreatic fistula: A matched-pairs analysis. Pancreatology, 2011; 11(5): 516-24

30. Nahm CB, Brown KM, Townend PJ et al: Acinar cell density at the pancreatic resection margin is associated with post-pancreatectomy pancreatitis and the development of postoperative pancreatic fistula. HPB (Oxford), 2018; 20(5): 432-40

31. Nahm CB, de Reuver PR, Hugh TJ et al: Intra-operative amylase concentration in peri-pancreatic fluid predicts pancreatic fistula after distal pancreatectomy. J Gastrointest Surg, 2017; 21(6): 1031-37 